\title{
Effects of Exogenous Melatonin on Antioxidant Activity of Kiwifruit Leaves in Response to Drought Stress
}

\author{
Xuewen Zhao ${ }^{1, a, \#, ~ H u i ~ X i a ~}{ }^{1,2, b, \#}$, Jin Wang ${ }^{1,2, c}$, Xiulan Lv ${ }^{1,2, d}$ and Dong Liang ${ }^{1,2, e, *}$ \\ ${ }^{1}$ College of Horticulture, Sichuan Agricultural University, Chengdu, Sichuan, China \\ ${ }^{2}$ Institute of Pomology and Olericulture, Sichuan Agricultural University, Chengdu, Sichuan, China \\ a867396930@qq.com, bsusanxia_2001@163.com, c251040278@qq.com, d1933063229@qq.com, \\ eliangeast@sina.com \\ \#contributed equally author, ${ }^{*}$ corresponding author
}

Keywords: Kiwifruit; Exogenous melatonin; Drought stress; Antioxidant Activity

Abstract. In this study, the seedlings of kiwifruit were used as the research materials to investigate the effects of exogenous melatonin (MEL) on antioxidant activity in kiwifruit leaves in response to drought stress. The results showed that exogenous melatonin effectively decreased the MDA content of leaves of kiwifruit seedlings under drought stress, increased the content of DPPH, ABTS and FRAP, improved the antioxidant activity and enhanced the ability of scavenging ROS. The results also indicated that exogenous melatonin alleviated the damage of drought stress on kiwifruit seedlings and improved the plants stress resistance.

\section{Introduction}

The fruit of kiwifruit contains lots of vitamin $\mathrm{C}$ and has high nutritional value [1]. Melatonin firstly was found in mammals in 1958 [2]. In recent years, melatonin has been found in higher plants [3-5]. Abiotic stress is an important factor limiting crop yield, and drought stress is one of the major factors [6]. Plants subjected to drought stress on growth and other physiological processes are affected in different aspects, and even lead to death. Studies have shown that melatonin can relieve the damage of plants under drought stress [7]. At present, the effects of exogenous melatonin on the leaves of kiwifruit under drought stress are still very few. The aim of this study was to discuss the effects of exogenous melatonin on the antioxidant activity of kiwifruit seedlings under drought stress.

\section{Materials and Methods}

Materials and Treatment. The seeds of annual wild kiwifruit were placed at $4^{\circ} \mathrm{C}$ refrigerator for 60 days. After pregermination with heterotherm treatment at $4^{\circ} \mathrm{C} 10 \mathrm{~h} / 25^{\circ} \mathrm{C} 14 \mathrm{~h}$ for a week, the germinated seeds were sown in $25^{\circ} \mathrm{C}$ incubator for two weeks until they grew to two-ture-leaf stage. The seedlings were moved into plastic pots (diameter: $18 \mathrm{~cm}$; height: $23 \mathrm{~cm}$ ) filled with mixed soil consisting of peat substrate, pulverized coconut shell and perlite, with three seedlings per pot. We watered the seedlings at 2-d intervals with 1/2 Hoagland's nutrient solution from the two-true-leaf stage ( $\mathrm{pH}$ adjusted to $6.5 \pm 0.1$ with diluted $\mathrm{HCl}$ or $\mathrm{NaOH}$ ).

Treatments began at 10-true-leaf stage, as follows: (1) Control (CK), plants were well-watered during the whole experimental time; (2) drought treatment (DR): seedlings were well-watered for 8 days, subsequently irrigation was withheld for up to 9 days; (3) melatonin and drought treatment (MTDR): seedlings were pretreated with $100 \mu \mathrm{M}$ melatonin solution for 4 times, two days once, then irrigation was withheld for up to 9 days. Each treatment was repeated three times. Sample their middle leaves (from five to eight per plant) after $0,3,6$, and $9 \mathrm{~d}$ of drought treatment. All seedlings leaves were immediately frozen in liquid nitrogen and stored at $-80^{\circ} \mathrm{C}$.

Physiological Indexes. Malonaldehyde (MDA) determination method adopted $\mathrm{Li}$ [8]. $\mathrm{DPPH}$ free radical scavenging capacity $(\mathrm{DPPH}), \mathrm{ABTS}^{\circ+}$ free radical scavenging capacity (ABTS) and iron ion reduction capacity (FRAP) were measured by Du [9]; The above indicators were set three times, and calculated the average value. 
Data Handling. Software Excel 2010 was used to calculate the test data and plot. Statistical analysis was performed using software Excel SPSS.

\section{Result and Analysis}

MDA Content. The MDA content in the leaves of DR and MTDR treatments increased significantly, especially from 6 to 9 days and peaked at 9d were 21.78 and $15.05 \mathrm{nmol} \cdot \mathrm{g}^{-1} \mathrm{FW}$ respectively, when compared with $\mathrm{CK}$ increased by $181.95 \%$ and $61.65 \%$ respectively. However, the MDA content in the leaves of MTDR group was lower than that of DR group, especially at 9d (Fig.1).

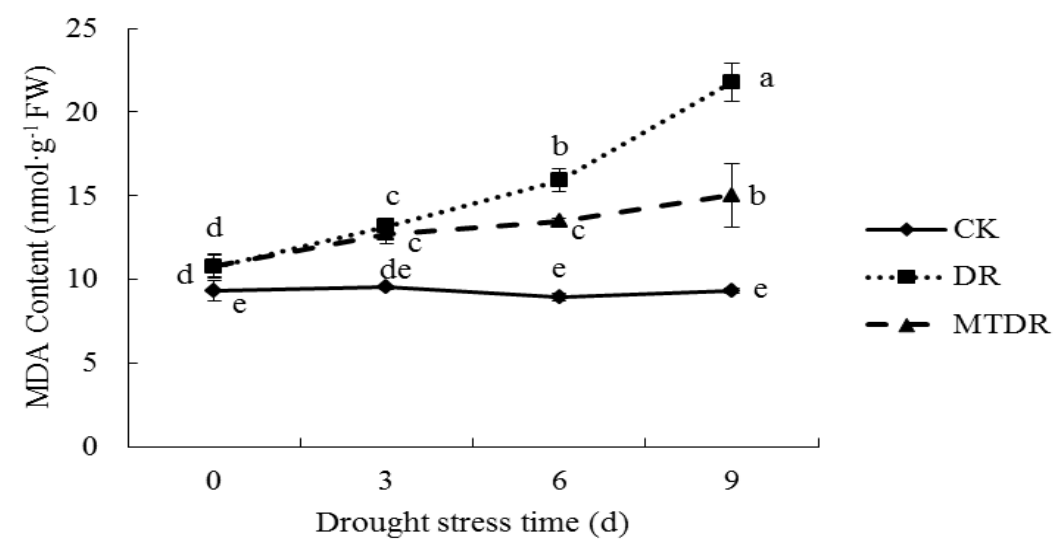

Fig. 1. Effect of exogenous melatonin on MDA content in leaves of kiwifruit seedlings under drought $\mathrm{stress}\left(\mathrm{nmol} \cdot \mathrm{g}^{-1} \mathrm{FW}\right)$. Note: Data with the different letters indicate the difference is significant $(\mathrm{P}<0.05)$.

DPPH Content. As seen from Fig.2, with the prolongation of drought stress, the DPPH content of DR increased and then decreased. At 0-6d, the DPPH content of DR increased gradually, and reached a maximum value of $3.14 \mu \mathrm{mol} \cdot \mathrm{g}^{-1} \mathrm{FW}$, then decreased gradually. The DPPH content of MTDR showed an upward trend, and was always higher than that of CK and DR. The results indicated that exogenous melatonin treatment improved the DPPH content of kiwifruit seedlings drought copper stress.

ABTS Content. As shown from Fig.3, after drought stress treatment, the ABTS content of DR and MTDR all were increased all over the time, which were higher than that of CK at 3-9d, and peaked at 9d, to $14.55 \mu \mathrm{mol} \cdot \mathrm{g}^{-1} \mathrm{FW}$ and $14.67 \mu \mathrm{mol} \cdot \mathrm{g}^{-1} \mathrm{FW}$, respectively. At 6-9d, the ABTS of DR and MTDR increased significantly. Therefore, at 6-9d, the ABTS content of MTDR were higher than that of DR. It could be concluded that drought stress increased the ABTS content of the leaves of kiwifruit seedlings, as well as exogenous melatonin increased the ABTS content of kiwifruit under copper stress, so then enhanced the antioxidant activity of plants.

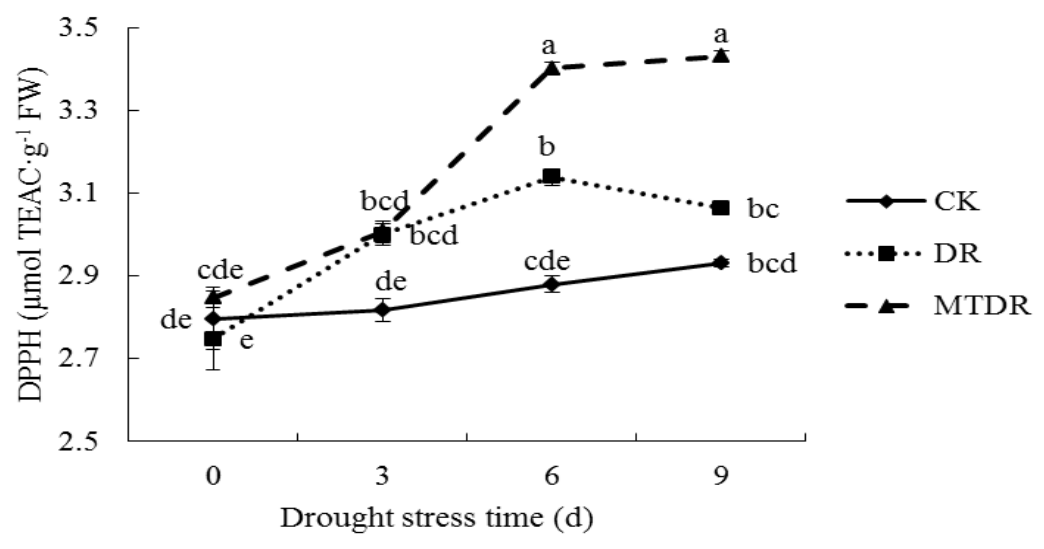

Fig. 2. Effects of exogenous melatonin on the DPPH content in leaves of kiwifruit seedlings under drought stress ( $\mu$ mol $\left.\mathrm{TEAC} \cdot \mathrm{g}^{-1} \mathrm{FW}\right)$. Note: Data with the different letters indicate the difference is significant $(\mathrm{P}<0.05)$. 


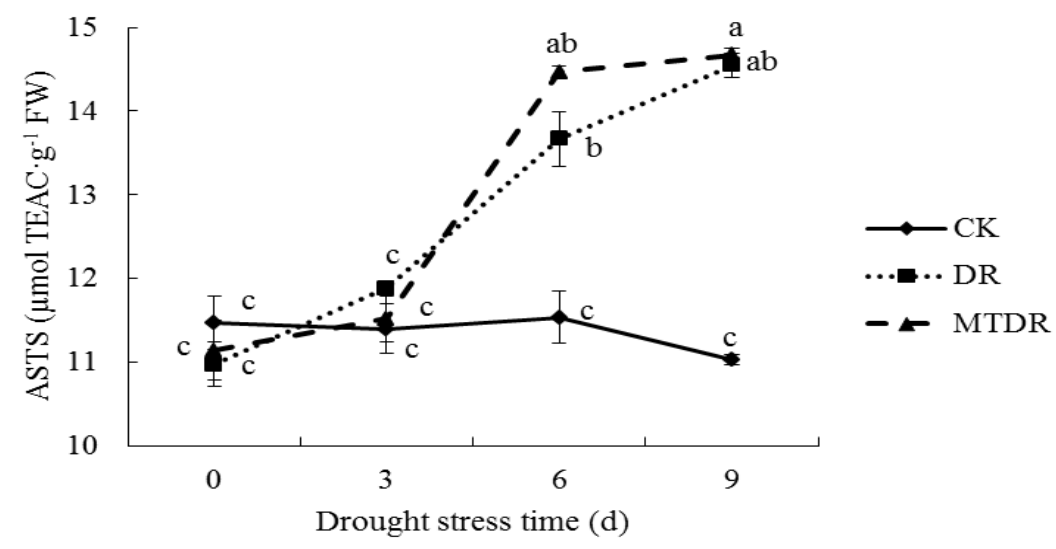

Fig. 3. Effects of exogenous melatonin on the ABTS content in leaves of kiwifruit seedlings under drought stress ( $\mu$ mol TEAC $\left.\cdot \mathrm{kg}^{-1} \mathrm{FW}\right)$. Note: Data with the different letters indicate the difference is significant $(\mathrm{P}<0.05)$.

FRAP Content. Fig. 4 is the change of the FRAP content of kiwifruit seedlings in the process of drought stress. When drought stress 0d, the change of the FRAP of each treatment was not obvious. With the prolongation of drought stress, the FRAP content of DR and MTDR showed an upward trend, which peaked at 9d, to $20.98 \mu \mathrm{mol} \cdot \mathrm{g}^{-1} \mathrm{FW}, 22.18 \mu \mathrm{mol} \cdot \mathrm{g}^{-1} \mathrm{FW}$. At 3-9d, the FRAP content of DR and MTDR always were higher than that of CK. The FRAP content of MTDR was always higher than DR, at $6 \mathrm{~d}$ and 9d. The results indicated that drought stress added the FRAP content of kiwifruit seedlings, and exogenous melatonin treatment improved the FRAP content of kiwifruit seedlings under drought stress, and enhanced plant antioxidant ability to heavy drought stress.

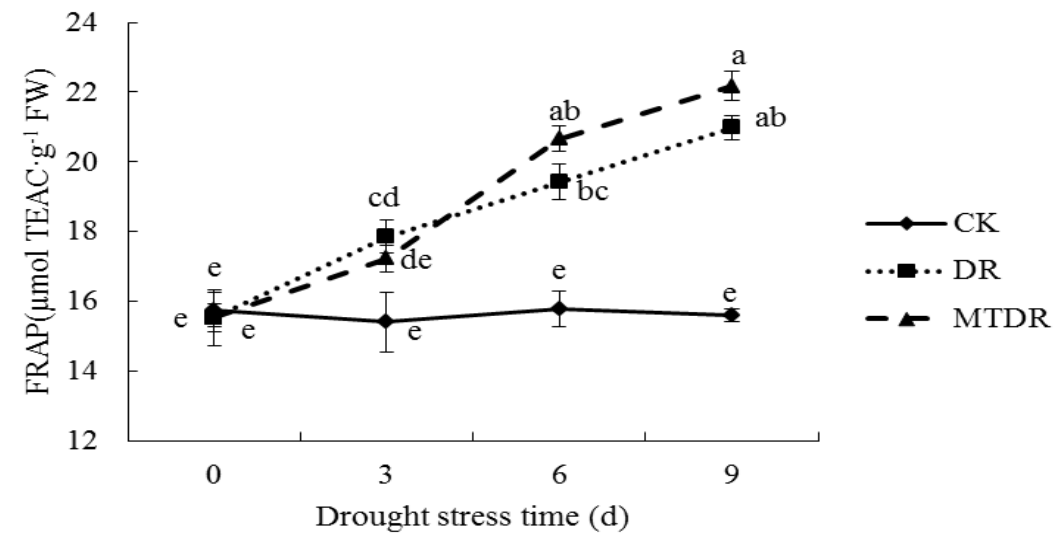

Fig. 4. Effects of exogenous melatonin on the FRAP content in leaves of kiwifruit seedlings under drought stress ( $\mu$ mol TEAC $\left.\cdot \mathrm{kg}^{-1} \mathrm{FW}\right)$. Note: Data with the different letters indicate the difference is significant $(\mathrm{P}<0.05)$.

\section{Conclusions}

Drought is an important factor affecting the growth and development of crops, especially at seedling Stage [10-12]. Under drought stress, plant cells initiate a series of scavenging mechanisms of ROS that produce antioxidants, which can remove the excessive ROS, thereby alleviating oxidative stress. MDA reflects cell damage degree, therefore it can be related to the drought resistance of plants [13]. DPPH, ABTS, FRAP reflect the antioxidant activity of plants. The results show that exogenous melatonin treatment can increase the DPPH, ABTS and FRAP content of kiwifruit seedlings under drought stress, improve the ability to scavenge free radicals to enhance the antioxidant activity, and then advance the ability of plant adversity resistance. It is consistent with the results of Wang et al. [14] on apple leaves and Ye et al. [15] on wheat seedlings. 


\section{Acknowledgements}

This work was supported by fund of the Science and Technology Department of Sichuan Province (2016NZ0105).

\section{References}

[1] J.V. Possingham: Scientia Horticulture Vol. 1-2 (1991), p. 171.

[2] A.B. Lerner, J.D. Case and Y. Takahashi: Am Chen Soc. Vol. 80 (1958), p. 2592.

[3] G.A. Bubenik: Gastrointestinal melatonin: Digestive Diseases and Sciences Vol. 47 (2002), p. 2347.

[4] J. Zagajewski, D. Drozdowicz and I. Brzozowska: Journal of Physiology and Pharmacology Vol. 63 (2012).

[5] R. Dubbels, R.J. Reiter and E. Klenke: Journal of Pineal Research Vol. 18 (1995), p. 30.

[6] J.K. Zhu: Annual Review of Plant Biology Vol. 53 (2002), p. 271.

[7] C.Q Jiang and Z.L. Zu: Biotechnology Information Vol. 31 (2015), p. 49, 50 (In Chinese).

[8] H.S. Li: Principles and Techniques of Plant Physiological and Biochemical Experiment (Higher Education Press, China 2000).

[9] G.R. Du: Analysis of antioxidant activity and antioxidant activity of kiwifruit, persimmon and apple fruits (Northwest A\&F University, China 2009).

[10]M.T. Harrison, F. Tardieu, Z. Dong, et al: Global Change Biology Vol. 20 (2014), p. 878.

[11]D.B. Lobell, M.J. Roberts, W. Schlenker, et al: Science Vol. 344 (2014), p. 519.

[12]C.L. Cao, S.X. Li: Acta Agriculturae Nucleatae Sinica Vol. 18 (2004), p. 404 (In Chinese).

[13]Q. Sun, N.H. Cai and S. Chen: Forum Vol. 18-22 (2016), p. 21 (in Chinese).

[14]P. Wang, X. Sun, C. Li, et al: Journal of Pineal Research Vol. 54 (2013), p. 299.

[15]J. Ye, X.P. Deng, S.W. Wang, et al: Journal of Triticeae crops Vol. 35 (2015), p. 1282 (In Chinese). 